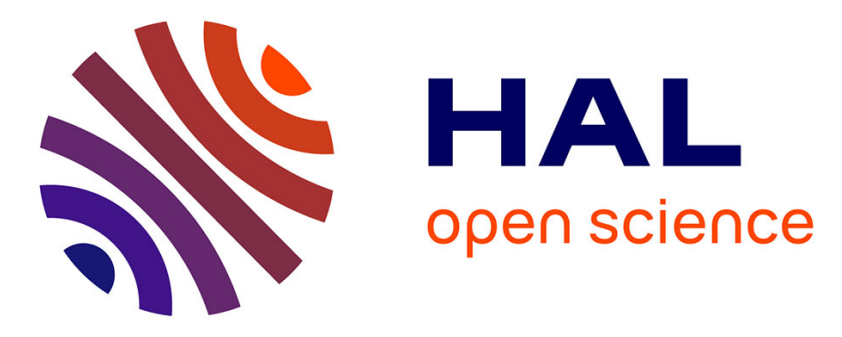

\title{
Data collection and preliminary results on turbulence characterisation and mitigation techniques
}

\author{
Marie-Thérèse Velluet, Christopher Bell, Jean-François Daigle, Judith Dijk, \\ Szymon Gladysz, Andrey Kanaev, Andrew Lambert, Daniel Lemaster, Guy \\ Potvin, Mikhail Vorontsov
}

\section{To cite this version:}

Marie-Thérèse Velluet, Christopher Bell, Jean-François Daigle, Judith Dijk, Szymon Gladysz, et al.. Data collection and preliminary results on turbulence characterisation and mitigation techniques. SPIE Security \& Defence 2019, Sep 2019, Strasbourg, France. 10.1117/12.2533821 • hal-02408796

\section{HAL Id: hal-02408796 https://hal.science/hal-02408796}

Submitted on 13 Dec 2019

HAL is a multi-disciplinary open access archive for the deposit and dissemination of scientific research documents, whether they are published or not. The documents may come from teaching and research institutions in France or abroad, or from public or private research centers.
L'archive ouverte pluridisciplinaire HAL, est destinée au dépôt et à la diffusion de documents scientifiques de niveau recherche, publiés ou non, émanant des établissements d'enseignement et de recherche français ou étrangers, des laboratoires publics ou privés. 


\title{
Data collection and preliminary results on turbulence characterisation and mitigation techniques
}

\author{
M.-T. Velluet ${ }^{* a}$, C. Bell ${ }^{\text {b }, ~ J .-F . ~ D a i g l e ~}{ }^{\mathrm{c}}$, J. Dijk $^{\mathrm{d}}$, S. Gladysz ${ }^{\mathrm{e}}$, A. Kanaev ${ }^{\mathrm{f}}$, A. Lambert $^{\mathrm{g}}$, D. Lemaster ${ }^{\mathrm{h}}$, \\ G. Potvin ${ }^{\mathrm{c}}, \mathrm{M}$. Vorontsovi \\ aOnera, The French Aerospace Lab, 92322 Châtillon, France; \\ ${ }^{b}$ Dstl Fort Halstead, Sevenoaks, Kent, TN14 7BP, UK \\ ${ }^{c}$ DRDC - Valcartier Research Center, 2459 de la Bravoure Road, Quebec City, Québec, Canada \\ 'TNO, PO Box 96864, NL-2509 JG The Hague, The Netherlands; \\ ${ }^{\mathrm{e}}$ Fraunhofer Institute of Optronics, System Technologies and Image Exploitation, Gutleuthausstr. 1, \\ 76275 Ettlingen, Germany; \\ ${ }^{\mathrm{f}} \mathrm{NRL}, 4555$ Overlook av., Washington DC 20375, \\ ${ }^{\mathrm{g}}$ UNSW Canberra, Canberra, Australia, \\ ${ }^{\mathrm{h}}$ Air Force Research Laboratory, 2241 Avionics Circle, Wright-Patterson AFB, OH, USA 45433, \\ ${ }^{\mathrm{i}}$ Intelligent Optics Laboratory, School of Engineering, University of Dayton, \\ 300 College Park, Dayton, OH, 45469-2951, USA
}

\begin{abstract}
In the framework of NATO task group SET 226 on turbulence mitigation techniques for OA systems, a trial was conducted in the premises of RDDC-Valcartier, using indoor and outdoor facilities in September 2016. Images data sets were collected under various turbulence conditions, both controllable (indoor) and natural (outdoor). The imagery of this trial was used in the Grand Challenge, where different experts were asked to process identical input data with state-ofthe-art algorithms. The trial also provided a data-base to validate theoretical and numerical models.

The paper will give an overview of the experiment set-up (target, sensors, turbulence screens generators...) and present some preliminary results obtained with the collected data in terms of effectiveness of image processing techniques, new methods for turbulence characterisation, modelling of laser beam propagation.
\end{abstract}

Keywords: Turbulence, propagation, experiments, image processing, laser beam delivery, adaptive optics

\section{INTRODUCTION}
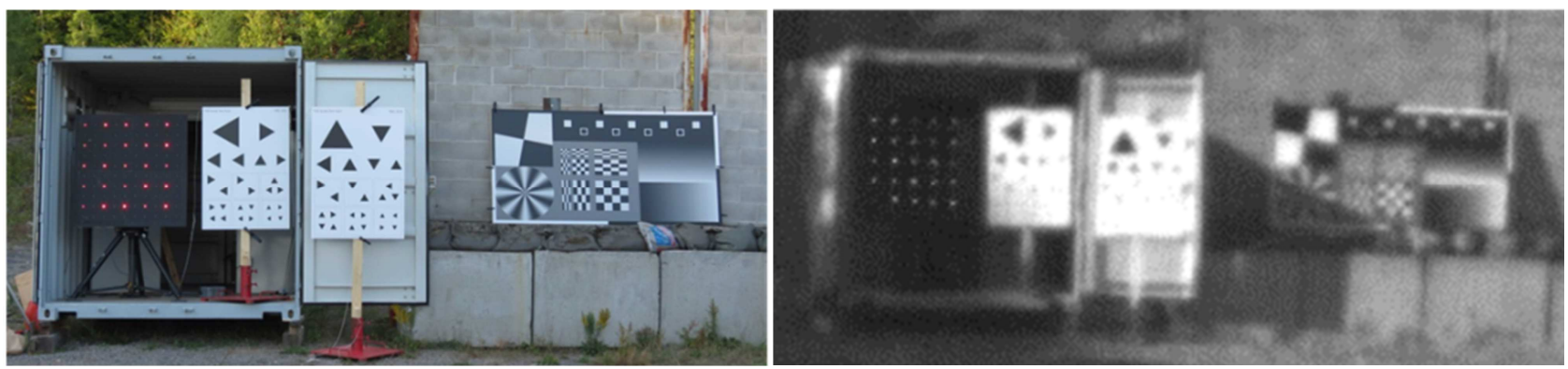

Figure $1:$ Left target area; right one target collected image at $2.5 \mathrm{~km}$.

High performance optical systems suffer from atmospheric turbulence present near the ground that limits their range and resolution. Because the need of target recognition, tracking or threat defeat at longer distance increases, Electro-optics (EO) and laser systems are more and more sensitive to turbulence effects. Illustrations of turbulence effects are presented in the 
figures below. The decrease of resolution can be seen in Figure 1 and the decrease of focused beam quality with the increase of turbulence strength expressed by $\mathrm{C}_{\mathrm{n}}{ }^{2}$ in Figure 2 .
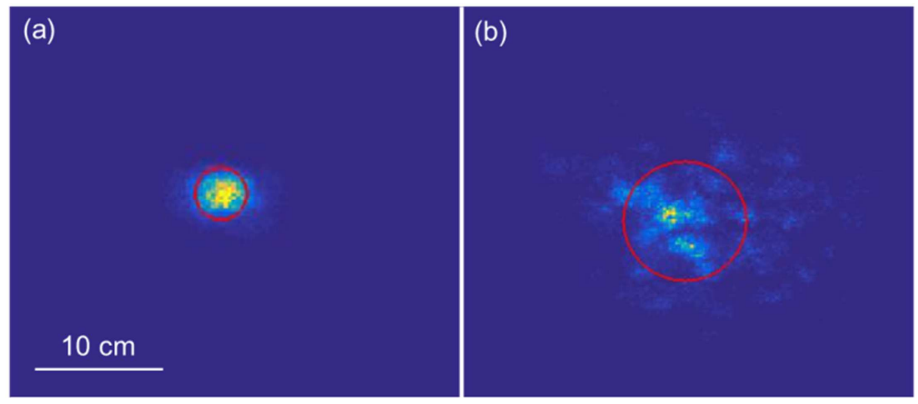

Figure 2 : Beam focusing at $2.5 \mathrm{~km}$ for (a) $\mathrm{C}_{\mathrm{n}}{ }^{2}=10^{-15} \mathrm{~m}^{-2 / 3}$ and (b) $\mathrm{C}_{\mathrm{n}}^{2}=7.5 \times 10^{-14} \mathrm{~m}^{-2 / 3}$.

Turbulence near the ground depends strongly on many variables, including sunlight, soil nature and humidity of the air. Over long distances, a great variability of environmental conditions can be found. This leads to a large spatial as well as temporal variability of turbulence conditions and lead to a varying impact and degradation level on the EO and laser (EOL) system performance. A better knowledge of turbulence strength and its distribution along the line of sight (LOS) can help to implement the most relevant turbulence mitigation techniques and to assess system performance models.

To overcome turbulence-induced limitations, mitigation techniques can be applied. Adaptive Optics (AO) systems are more efficient for laser beam delivery systems, while data processing is more suitable for visible/IR imaging sensors. However, higher the turbulence between target and receiver planes, lower the efficiency of these techniques.

The objective of the NATO SET 226 group is to improve the knowledge on turbulence features (strength, statistics, distribution ...) and the impact on EOL systems performance in order to propose adequate mitigation techniques and then test them. Here we describe the experiments which were conducted in the framework of NATO group SET-226 and present some interesting preliminary results.

\section{EXPERIMENTAL SET-UP}

A joint NATO trial was conducted at the premises located in DRDC - Valcartier Research Centre, located $20 \mathrm{~km}$ from Quebec City, Canada. Two distinct test sites were selected to perform the different experiments: a 220 m indoor range with allowing for systematic measurements in controlled and reproducible conditions and a $2.5 \mathrm{~km}$ outdoor range for real-world conditions.

Assessing the impact of atmospheric turbulence on EOL systems requires extensive testing at various ranges. An indoor environment allows control of, to a certain extent, individual parameters of the atmosphere, while outdoor ranges provide a good insight into the impact of realistic and dynamic weather conditions. In an indoor environment, the quasi-absence of turbulence can be circumvented by adding controlled heat sources along the path to generate artificial turbulence. The drawbacks of such a facility include a limited engagement range, and the lack of transverse wind profiles.

As compared to indoor tunnels, outdoor ranges generally offer significantly longer paths. No control is possible over the test conditions; however it is possible to record strong correlations in between the EOL system performance and specific weather and turbulence parameters. A short propagation range increases the probability of uniformity of conditions (turbulence, humidity, wind) along the entire propagation path while longer ranges usually exhibit a stronger variability of atmospheric parameters along the path due to the increased possibility of variations in beam height, wind conditions and terrain (e.g grass, roads or rivers).

\section{$2.1250 \mathrm{~m}$ indoor range}

The Aero-ballistic Range test site consists of a $250 \mathrm{~m}$ long indoor firing range located on the southern site of DRDCValcartier. The corridor, has been (and still remains) a test ground to characterize the ballistics and aerodynamics of projectiles used by the CAF. This fully equipped tunnel is also an ideal place to perform systematic EOL experiments in a controlled environment. An aerial view of the test site is presented in Figure 3(a). Figure 3(b) shows the interior of the tunnel which is fully equipped with cameras and imaging screens for characterization of the ballistics of various projectiles. 

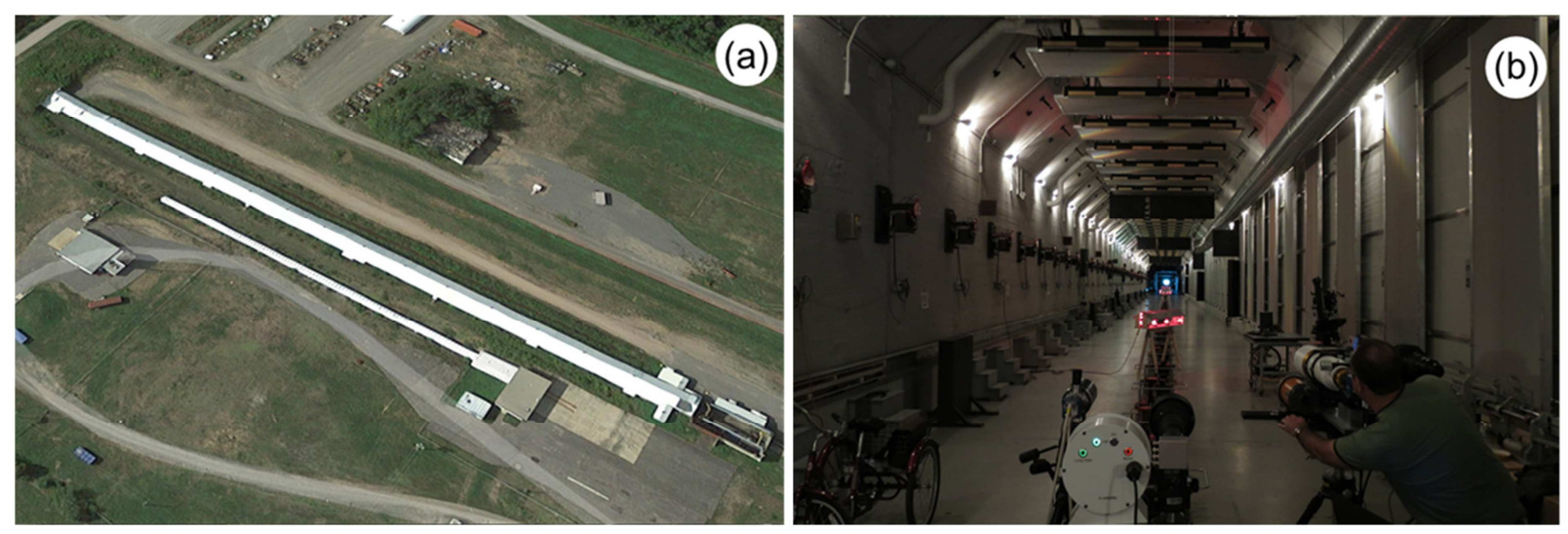

Figure 3: (a) Aerial of the $250 \mathrm{~m}$ long Aero-Ballistic Range firing site. (b) Interior of the Aero-Ballistic Range. The corridor is fully equipped with cameras used to characterize the ballistics and aerodynamics of projectiles.

\section{Description and characterization of the Turbulence Generators}

The turbulence generators consisted of six 250 Watt bulbs placed under a $4 \mathrm{~cm}$ by $183 \mathrm{~cm}$ vent formed from aluminum sheet, as depicted in Figure 4. The bulbs were arranged in two banks of three which could be individually turned on or off and the bulbs' temperature could be controlled using a rheostat. There were five sets in total.

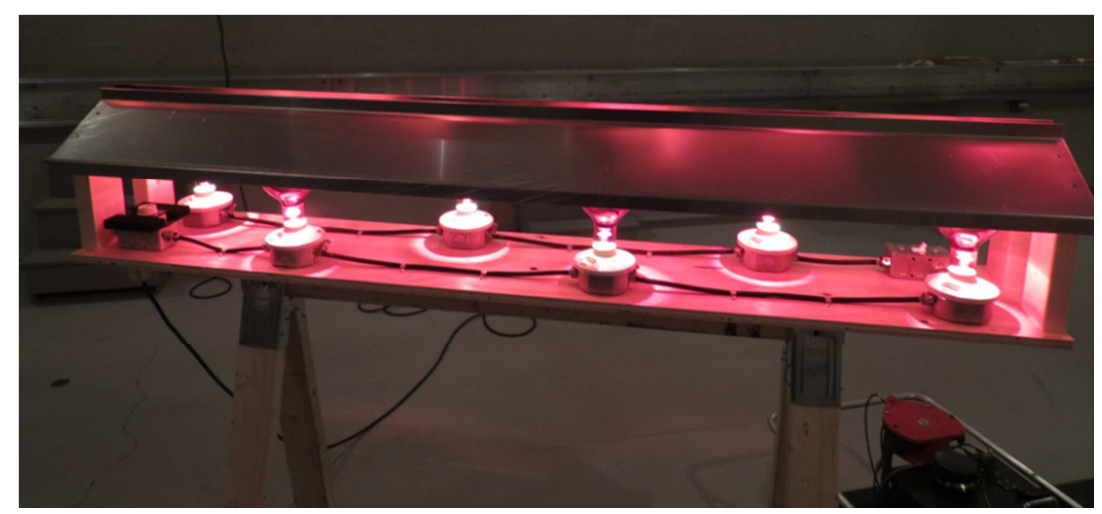

Figure 4: A turbulence generator consisting of six 250 Watt bulbs under an aluminium vent that is $4 \mathrm{~cm}$ wide and $183 \mathrm{~cm}$ long.

The turbulence generators were placed under the line of sight of the various cameras and lasers to produce a screen of optical turbulence. For some of the measurements the turbulence generators were placed at an angle across the beam to increase the turbulent path-length. Also, for some measurements, so that all the targets could be imaged simultaneously, two screens were placed next to each other, one in front of each target.

Table 1: Estimates from thermocouples measurements of outer scale, CT2 and $\mathrm{C}_{\mathrm{n}}{ }^{2}$.

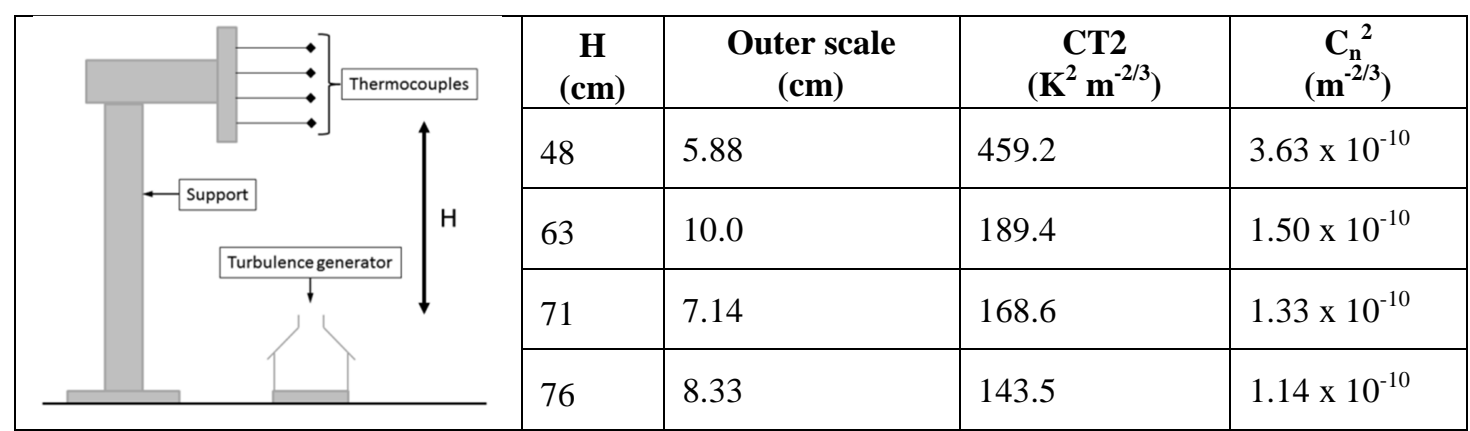


The turbulence generated by these devices was characterized using both optical means and temperature measurements from thermocouples. In this later case, turbulence feature has been derived and reported in Table 1. The turbulence strength is quite high, a few $10^{-10} \mathrm{~m}^{-2 / 3}$, which leads to Fried parameter ( $\left.\mathrm{r} 0\right)$ lower than $2 \mathrm{~cm}\left(2 \mathrm{~cm}\right.$ at $\lambda=0.6 \mu \mathrm{m}, \mathrm{C}_{\mathrm{n}}^{2}=10^{-10} \mathrm{~m}^{-2 / 3}$ and four screens at 10,110, 165 and $210 \mathrm{~m}$ from the target). This value is representative of the following case: propagation distance $=2500 \mathrm{~m}, \mathrm{C}_{\mathrm{n}}{ }^{2}$ constant along the $\mathrm{LOS}=1.510^{-14} \mathrm{~m}^{-2 / 3}$ which can be considered as medium turbulence conditions.

\section{Targets}

There were two main targets used in the tunnel: a LED panel provided by AFRL and a projection screen on which various targets could be projected. The projector used was an EPSON PowerLite Pro Z9900WNL This had a 1280 by 800 screen resolution with an output of 9200 lumens.

Both still and moving images were projected onto the screen. The still images included test charts e.g. the US Air Force 1951, vertical or horizontal sinusoidal bars. In addition, there were also images of natural scenes. The LED panel consisted of a 10x10 array of individually controllable sources with a10 cm spacing between them. The wavelength of the LEDs was $627 \mathrm{~nm}$ with an output of $12 \mathrm{~cd}$ and an $8^{\circ}$ output angle. Sample images of these targets are shown in Figure 5; an image through turbulence is also presented for the horizontal bars case.
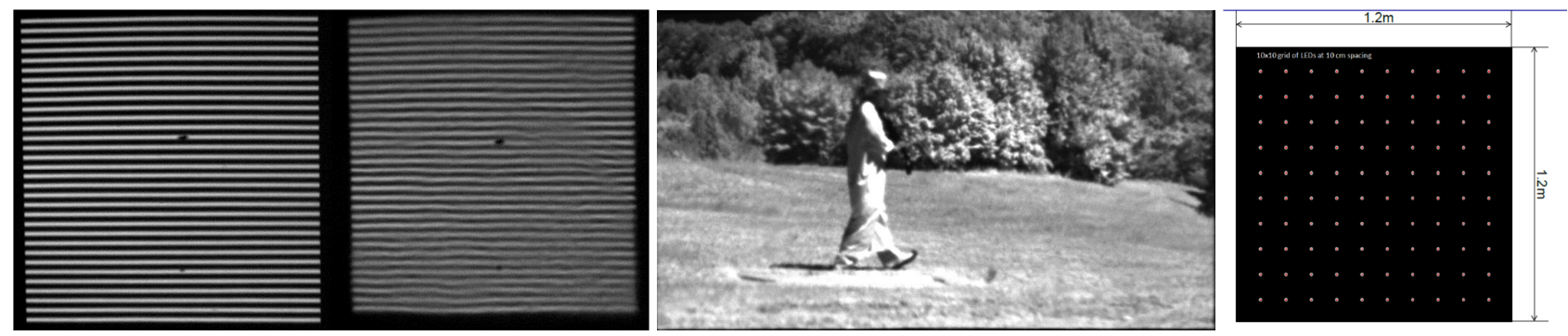

Figure 5: From left to right: Sample images of the horizontal sinusoidal bars from the pristine and the turbulent sequences, projected video of a man walking in a circle, LED array panel.

\section{$2.2 \quad 2500$ m Outdoor Range}

Outdoor testing was conducted on a $2.5 \mathrm{~km}$-long range available as part of DRDC infrastructures located at the nearby Canadian Forces Base in Valcartier. Figure 6(a) presents an aerial view of the outdoor range while Figure 6(b) presents the view of the range as seen from the position of the EOL systems.

During the experiments EO systems tested and the Canadian HEL platform were located at the lower-left extremity of the range depicted in Figure 6 (a) while all the targets and laser propagation diagnostics were installed at the other end of the range $2.5 \mathrm{~km}$ away. A garage was available to accommodate and protect all the EO sensors and related hardware from rain and humidity.

The trailer-mounted HEL system was installed beside the garage for the laser propagation tests and was aiming towards an interception screen installed in a sea container positioned at the other end of the range. 


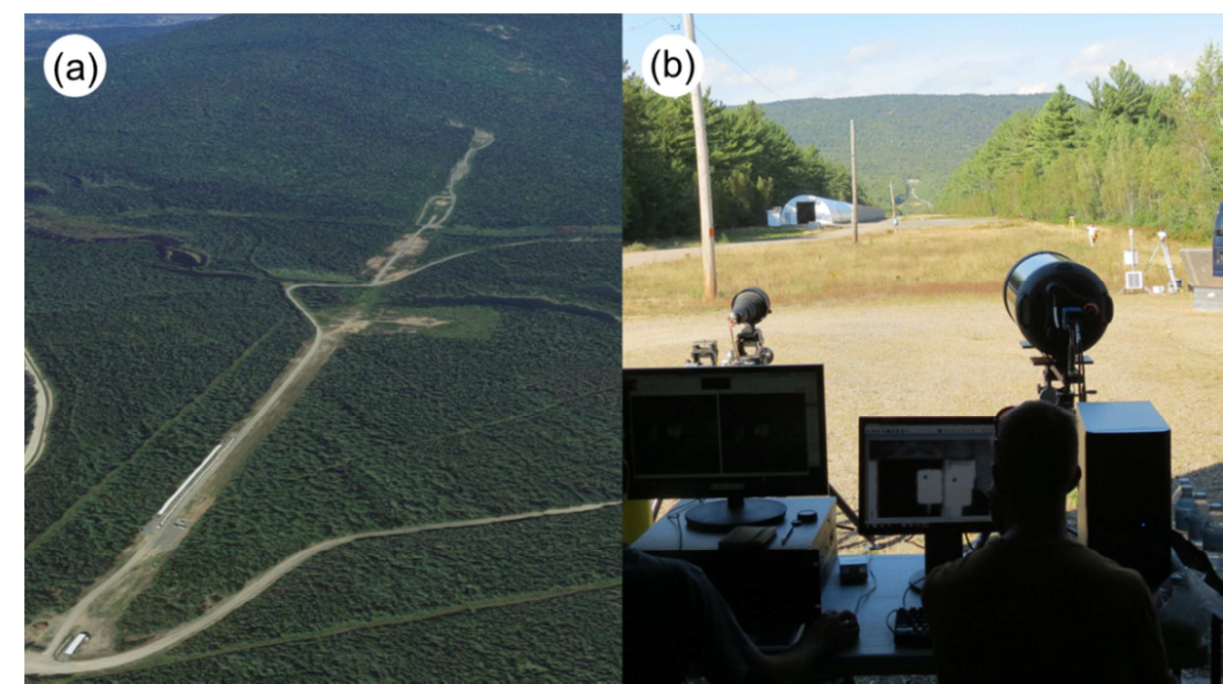

Figure 6: (a) Aerial of the $2.5 \mathrm{~m}$ outdoor range. (b) $2.5 \mathrm{~km}$ range view as seen from the interior of the garage accommodating EO sensors and related hardware.

\section{Instrumentation}

Different instruments were installed to monitor turbulence (2 scintillometers and a 2D sonic anemometer) and environmental conditions (a weather station).

\section{Targets}

There were two types of static test charts used: a standard USAF 1951 resolution chart and a triangle orientation discrimination (TOD) method test target. Also, the LED array was used again, but because of the increased range every other row and column was turned off, so producing a $5 \times 5$ array of LEDs.

In addition, live targets were used in the form of a moving van and a person walking for some of the serials.

\section{Description of cameras used}

For conventional imaging, there were four different visible (VNIR) cameras used for the image acquisition with different resolution and pixel size at the target (see Table 2) both for indoor and outdoor ranges. In front of the Baumer camera a plenoptic system was implemented.

Table 2: Cameras used for image acquisition

\begin{tabular}{|l|c|c|c|}
\hline \multirow{2}{*}{ Camera } & \multirow{2}{*}{ Resolution } & \multicolumn{2}{c|}{ Pixel size at the target $(\mathbf{c m})$} \\
\cline { 3 - 4 } & & Tunnel & Outdoor \\
\hline pco.edge 5.5 & $2560 \times 2140$ & 1.55 & 4.22 \\
\hline Mikrotron EOsens mini camera & $1280 \times 1024$ & 2.37 & 17.5 \\
\hline Optronis CamPerform CP80-3-M/C-540 & $640 \times 480$ & 2.20 & 25.4 \\
\hline AVT, Mako G-125C IRC PoE & $1,024 \times 964$ & 1.03 & 11.9 \\
\hline Baumer HXC13 & $1280 \times 1024$ & 3.12 & $36.0^{1}$ \\
\hline
\end{tabular}

\footnotetext{
${ }^{1}$ The projected pixel size quoted is if it were used as a conventional camera. As it was used as a plenoptic camera, the real projected pixel size would be different.
} 


\section{EXPERIMENTAL RESULTS}

\subsection{Turbulence characterization}

It is in principle possible to estimate path-integrated $C_{n}^{2}$ from image motion (angle-of-arrival method, Equation (1) [1]) but this approach is susceptible to vibrations, drifts and thermal effects on optics of the transmitter and the receiver.

$$
\left\langle\delta^{2}\right\rangle=2.91 D^{-1 / 3} \int_{0}^{L} C_{n}^{2}(z)\left(\frac{z}{L}\right)^{5 / 3} d z
$$

Measurement of differential image motion is not affected by any of these effects [2]. Assuming a pure Kolmogorov model, the angular correlation of the beam centroid of a grid of point sources can be expressed as a function of the $C_{n}^{2}$ profile [3] and is defined by eq. (2). For different position of the phase screens, this angular correlation has been studied. As presented in Figure 7 we have compared angular correlation given by eq. (2) assuming that the $C_{n}^{2}$ value is the same for each screen and between each phase screen the turbulence is very low and can be neglected.

$$
C_{x x}(i, j) \alpha \frac{d_{x}(i) d_{x}(j)}{\sigma_{x}(i) \sigma_{x}(j)}
$$
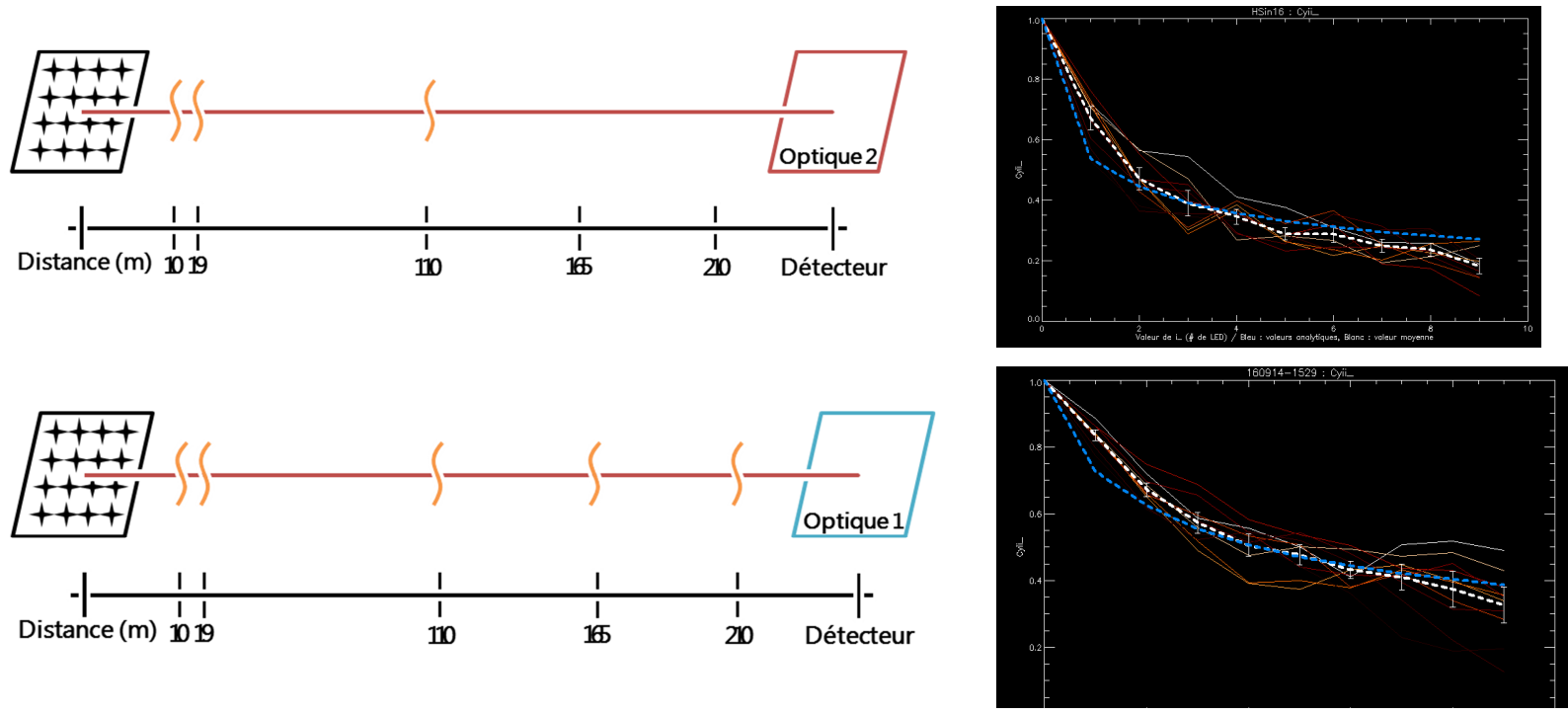

Figure 7: Left: configuration of the experiment; Right: $C_{x x}(i, j)$ as a function of the point sources position, white curve is the mean of the correlation figure obtained for each horizontal line and blue curve is given by the analytical expression [3].

We find a very good agreement between the measurement (line curve) and the theory (blue curve). This result is promising. In fact, the study of angular correlation should make it possible to estimate the relative strength of a few turbulence layers along the LOS.

Recordings of the LED array were also processed at Fraunhofer IOSB using the method outlined in Ref. [2]. Differential variances (structure functions) of motion were fitted with the Kolmogorov or the von Kármán models in order to extract $C_{n}^{2}$ and $L_{0}$ values. Processing of the same variances in the time domain is used to obtain the value of the transverse wind speed, or flow velocity for the case at hand. A "discrete" model of turbulence along the path can be easily inserted into the equations by replacing the integral $\int_{0}^{L} w_{\mathrm{s}}(z) C_{n}^{2}(z) d z$ with a sum $\sum_{i=1}^{m} w_{\mathrm{s}_{i}} C_{n_{i}}^{2} \Delta z_{i}$, where $w_{\mathrm{s}}(z)$ is the path weighting function for this experiment, $m$ is the number of heaters used and $\Delta z_{i}$ is the longitudinal extent of the turbulence "slab" created above each heater.

Figure 8 illustrates some of the results. The top panels show the agreement between the theoretical and the experimental differential motions for two chosen experiments (the agreement was similarly good for the other cases). The bottom left panel shows the extracted outer scale values and the bottom right panel shows the obtained values of flow velocity. 

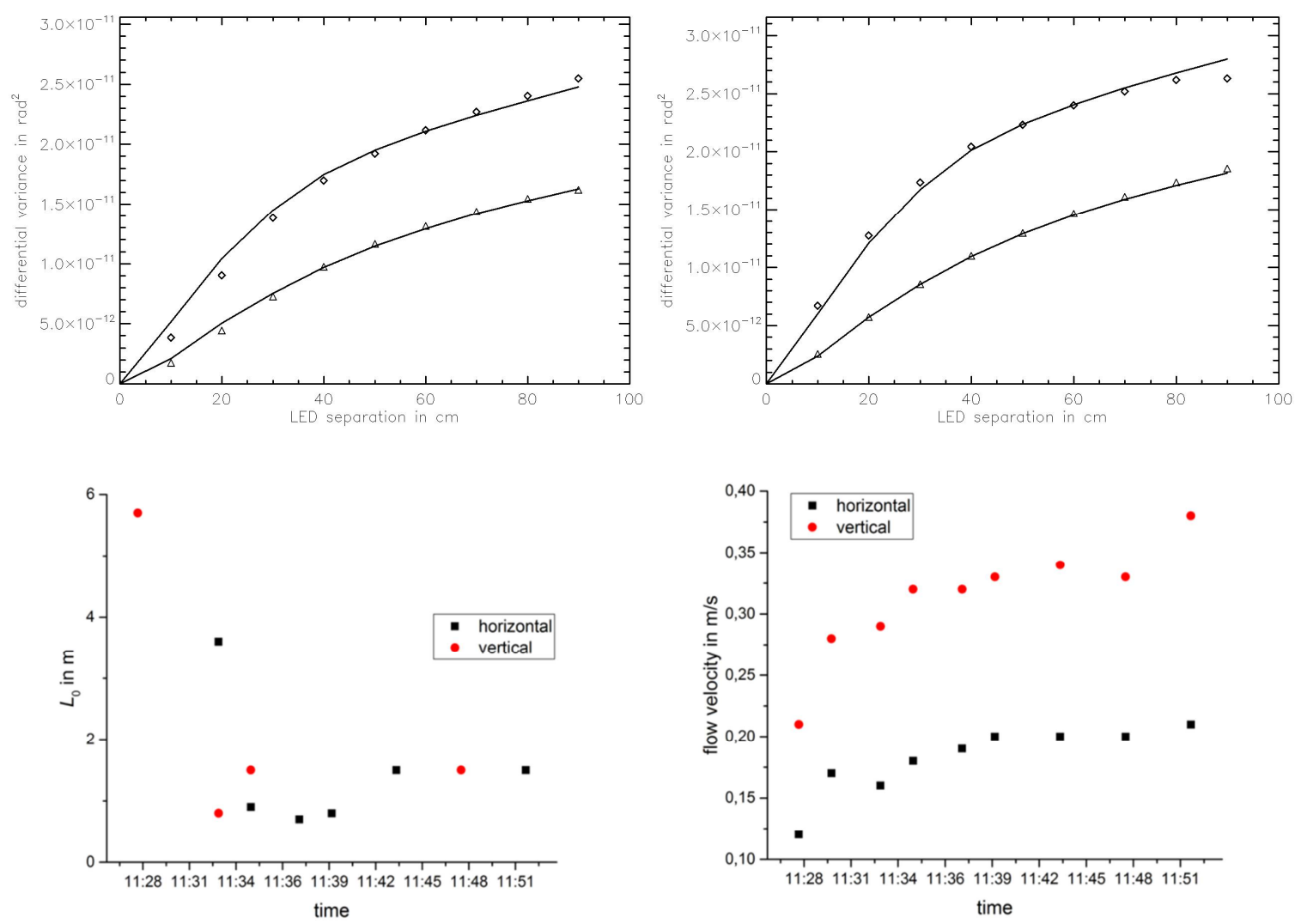

Figure 8: Top left: parallel and perpendicular differential motions, theory (solid lines) and experiment (symbols), data taken on September 15 at 11:28 local time. Top right: data taken on September 15 at 11:33 local time. Bottom left: outer scale values for the same time period. Top right: flow velocity measurements for the same time period.

\subsection{Laser propagation}

Thanks to the indoor facilities, the images of the beam in the focal plane can be recorded with different turbulence profile (number and location of the phase screens). The distortion of the beam takes a different structure depending on where in the path the phase screen generators are placed. Typical beam profiles are shown in Figure 9. For an ideal TEM00 beam in the absence of phase distortion we would expect a Gaussian focal image.

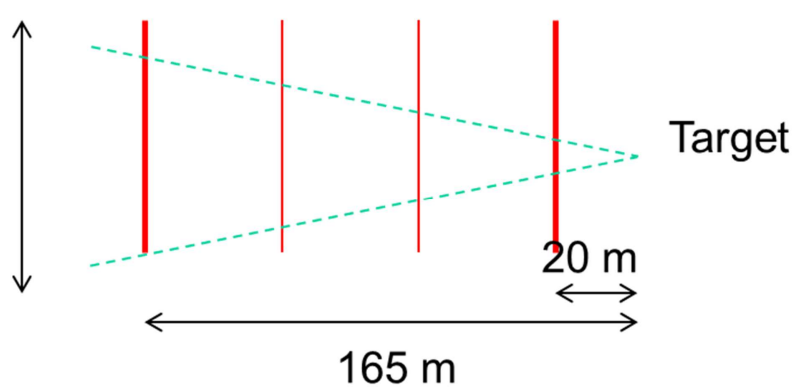

Phase screen at $20 \mathrm{~m}$ from the target

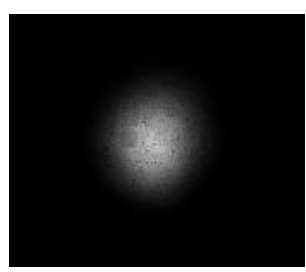

Phase screen at $165 \mathrm{~m}$ from the target

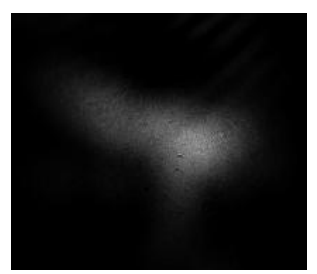

Figure 9: Influence of the turbulence on the laser beam quality.

Laser beam propagation has also been studied over longer distance (outdoor range) and the images of the beam recorded in the observation plane have compared with images obtained with the same propagation condition with numerical models. The size of the long exposure beam and the standard deviation of the beam motion were compared for both images. The results are presented in Figure 10 for different turbulence strengths. Experiments (back) and simulation 
(blue) are in good agreement except for very weak turbulence conditions where the experimental system is dominated by vibrations.

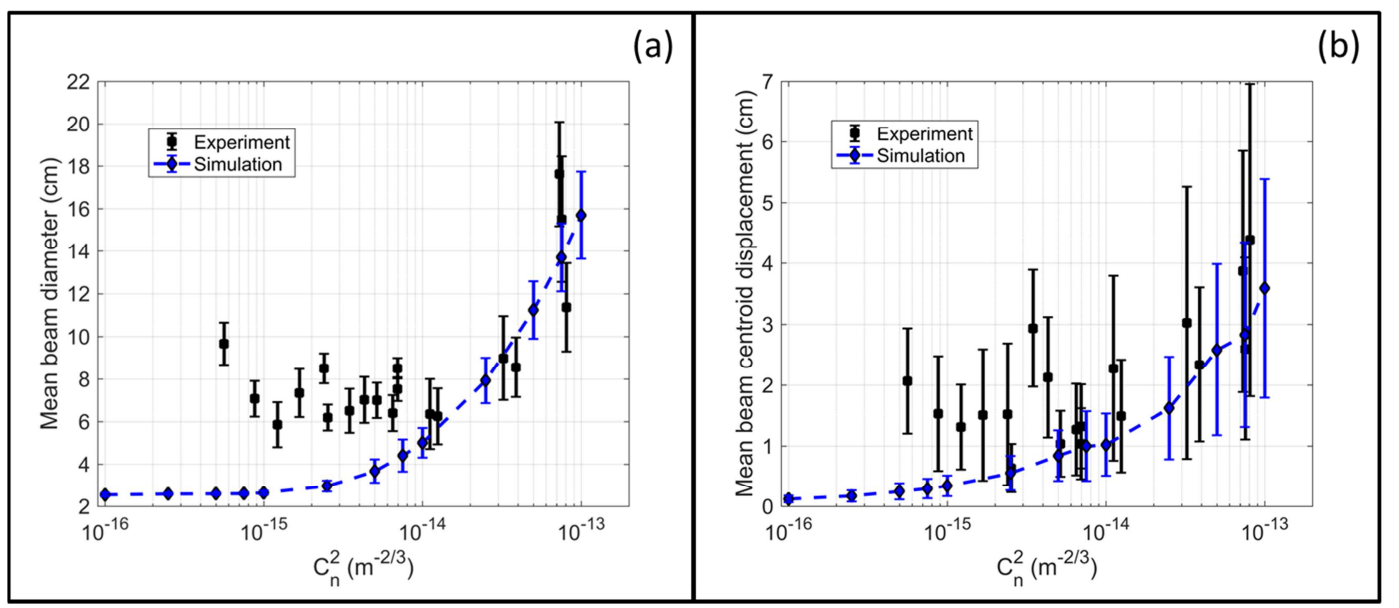

Figure 10: Statistical analysis of the mean beam spread (a) and the mean beam centroid displacement (b) measured as a function of $\mathrm{Cn} 2$.

\subsection{Plenoptic camera}

There are at least two kinds of plenoptic cameras: standard and focused plenoptic cameras[6]. In the standard plenoptic camera, the microlens array is coincident with the focal plane of the prime lens. The spatial resolution of the final image is equal to the number of lenses in the array and the number of 'directions' of the rays equals the number of pixels behind each microlens.
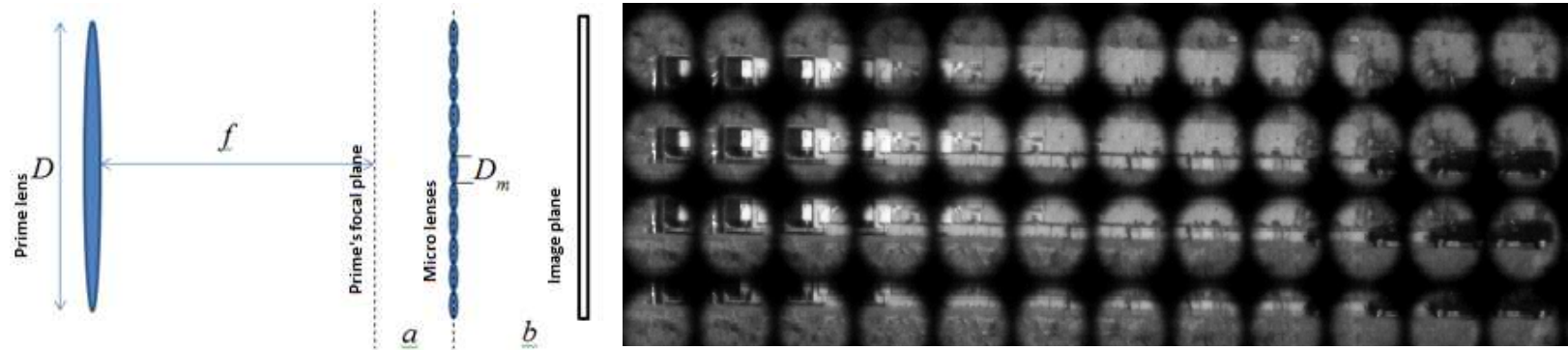

Figure 11: Left: principle of the focused plenoptic camera; Right: an example of recorded image.

In the focused plenoptic camera, the microlens array can be either in front or behind the focal plane of the prime [6]. In this camera, the microlens array was behind the prime lens's focal plane (Figure 11 - left). Each microlens refocuses a subsection of the prime's focal plane (i.e. the prime's image) onto a second focal plane, forming an array of micro images (Figure 11 - right). The spatial resolution of the focused plenoptic camera is b/a times the resolution of the sensor and the number of angular samples is $a / b$.

However, for each point in a micro image, all the rays come from a single circular patch on the lens of diameter $f D_{m} / a$. If this circular patch is smaller than $r_{0}$, then the major effect of turbulence on the image from the microlens will be shift and distortion.

Plenoptic camera was envisioned to measure phase and amplitude of disturbed wavefront of a laser beam after propagation through turbulence. The authors claim that it is potentially possible to reconstruct the turbulence volume with this type of sensor [6]. They also proposed this type of camera to overcome high image distortion given by conventional imaging systems [7]. Further studies must be carried-on in the future to evaluate limitations and advantages of plenoptic camera for target recognition through turbulence. 


\section{4 "Grand challenge"}

Previously there have only been a few turbulence mitigation algorithms compared simultaneously. It was decided to run a 'Grand Challenge' to compare the performance of as many algorithms as possible against the static USAF 1951 chart. This was done by inviting leading academic, military, and defense industry researchers to participate. As an enticement, results were to be shared anonymously across all participants. The restoration algorithms represented in the challenge included a mix of published and proprietary approaches. Among the published approaches, the bibliography contains a cross-section of applicable papers (References [6]-[19]). In this way, each participant would be able to evaluate their competition without risking anything if their submission compared poorly. For the SET-226 group, the outcome allows for a broad survey of the state-of-the-art from experts at very little cost. In total there were 14 different participants

\section{Data collection}

The static scene used in the restoration challenge includes a USAF 1951 resolution target, a $5 \times 5$ array of $20 \mathrm{~cm}$ spaced LEDs, a scintillometer, and the TNO triangle orientation/acuity target, among other things. The range from telescope to target was $2.54 \mathrm{~km}$ with a path-averaged refractive index structure parameter, $\mathrm{C}_{\mathrm{n}}{ }^{2}$, of $2.33 \mathrm{e}-15 \mathrm{~m}^{-2 / 3}$ as measured by the scintillometer. This scenario corresponds to a band-averaged coherence diameter of $0.071 \mathrm{~m}$. The telescope used in this test was a Celestron StarBright XLT with a focal length of $3.91 \mathrm{~m}$ and $0.356 \mathrm{~m}$ aperture ( $35 \%$ linear obscuration). The imagery was recorded using a PCO.edge camera with $6.5 \mu \mathrm{m}$ detectors (unfiltered silicon) operated with 2 ms integration time at 37 frames per second. The first frame in the image sequence is shown in Figure 12.

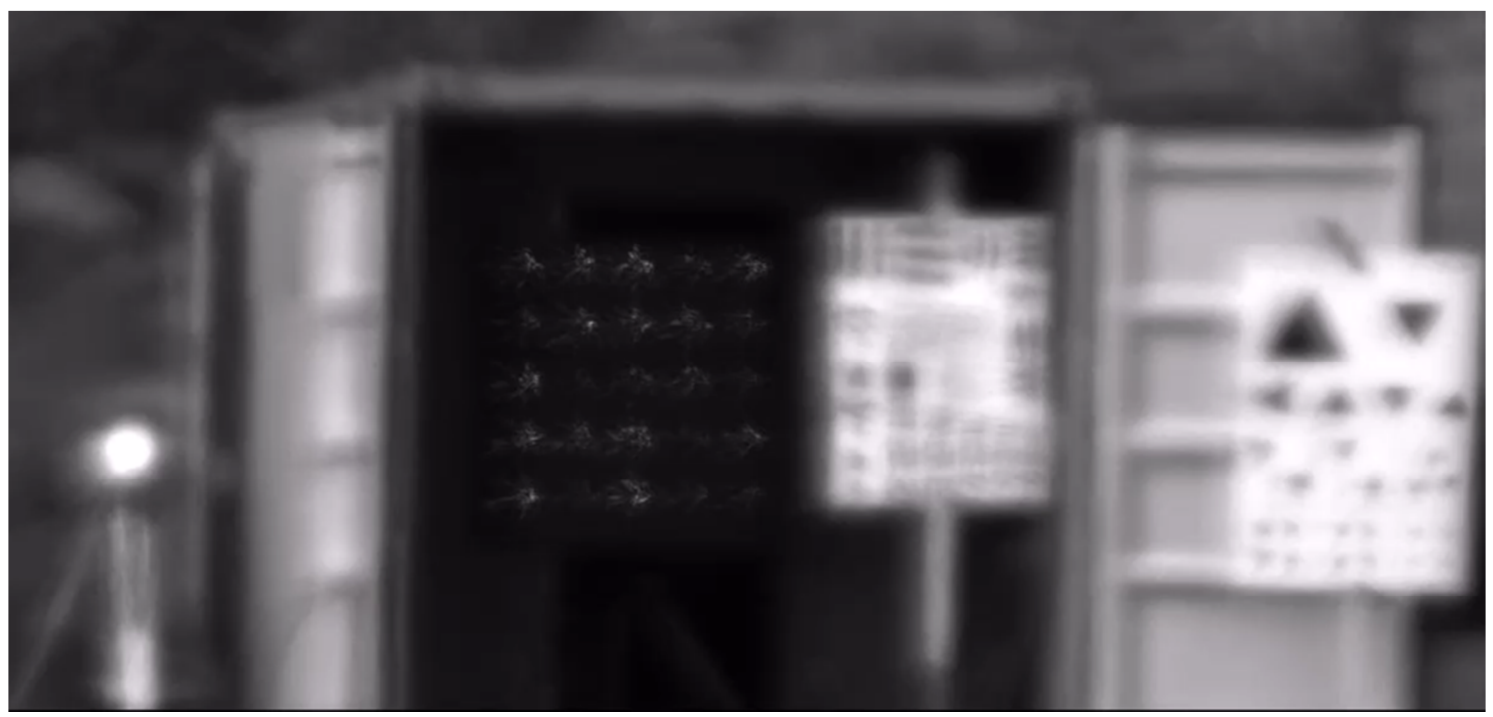

Figure 12: The first frame in the static target challenge.

The static target test was useful because comparison between restoration algorithms can be quantified in terms of limiting resolution. In the static target challenge, participants were asked to produce the best possible restoration given 200 sequential images of the turbulence degraded scene. No time limit was placed on the effort and the definition of "best possible restoration" was left open to individual interpretation. 

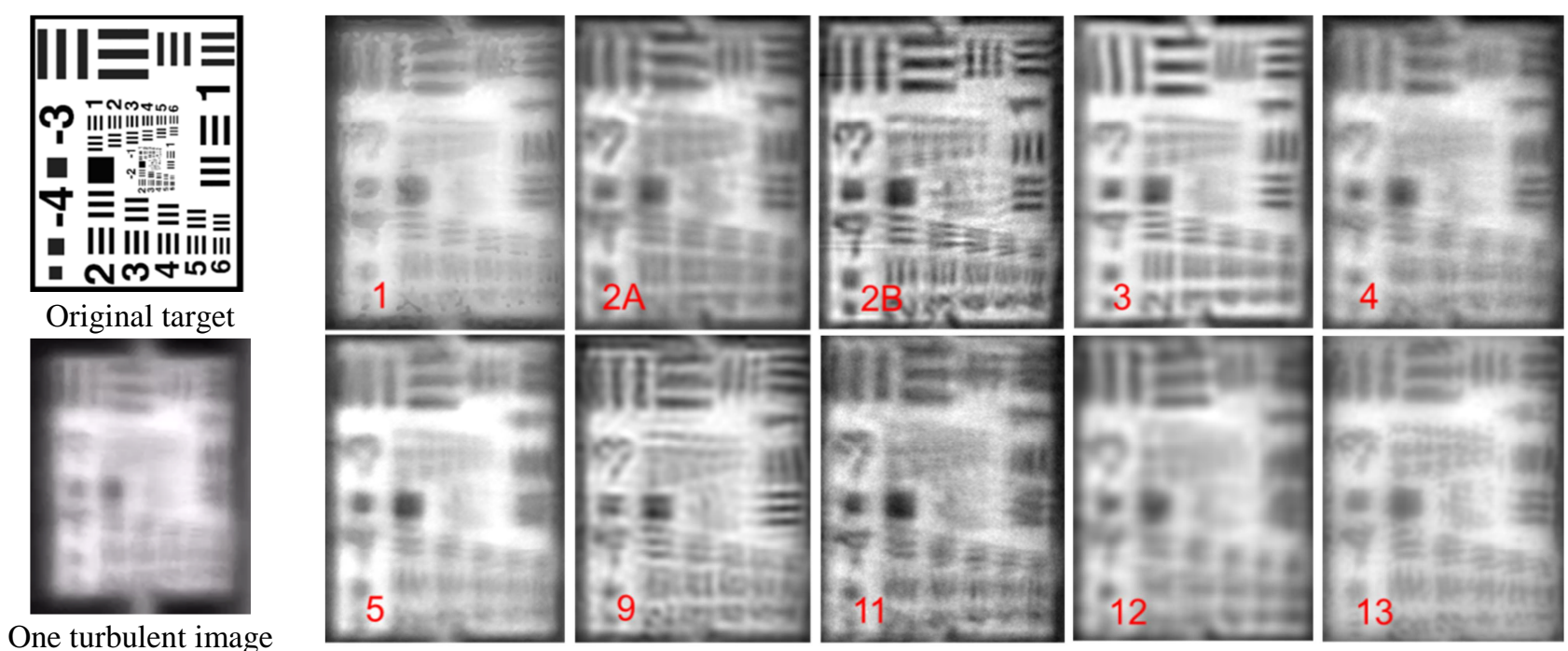

Figure 13: Left: original target and one turbulent image; left: Montage of static target restoration challenge results. Some results are not shown -8 is very similar 9 and 10 is not competitive.

\section{Scoring}

Restoration algorithm performance is quantified by determining the smallest resolvable bar pattern on the USAF 1951 test target in each restored submission. A bar pattern is resolved if all three horizontal and vertical bars are apparent. For the results shown in Figure 13, this determination was made by group consensus of the NATO SET-226 members during a meeting of the group (June 2017) at FFI in Kjeller, Norway. The results are reported in Table 3 as well the limited resolution in terms of line pairs/mrad.

Table 3: Summary of the static target challenge results.

\begin{tabular}{|c|c|c|c|c|c|c|c|c|c|c|}
\hline Method & 1 & $2 \mathrm{~A}$ & $2 \mathrm{~B}$ & 3 & 4 & 5 & 9 & 11 & 12 & 13 \\
\hline Resolved & G4 & G4 & G4 & G4 & G4 & G4 & G4 & G4 & G5 & G4 \\
\hline (Group/element) & $\mathrm{E} 5 / 6$ & $\mathrm{E} 2 / 3$ & E6 & $\mathrm{E} 4 / 5$ & E1 & E2 & E1 & $\mathrm{E} 1 / 2$ & E6 & E3/4 \\
\hline $\begin{array}{l}\text { Limiting Resolution } \\
\text { (line pairs / mrad) }\end{array}$ & 97.25 & 68.8 & 102.8 & 86.7 & 57.7 & 64.9 & 57.7 & 61.3 & 51.4 & 77.2 \\
\hline
\end{tabular}

Simple criteria were also used to evaluate the quality of the restored image for two resolutions (see Figure 14, left). These criteria are 1) noise = std in structure free area, 2) the Contrast-to-Noise-Ratio (CNR) measurement is to see if the bar patterns are still visible, and is expressed by $C N R=($ diff $) /$ noise where $\operatorname{diff}=\max -\min$. There were determined on the selected images of Figure 13 (right) and presented in Figure 14 (left for the large bars and right for the small bars).

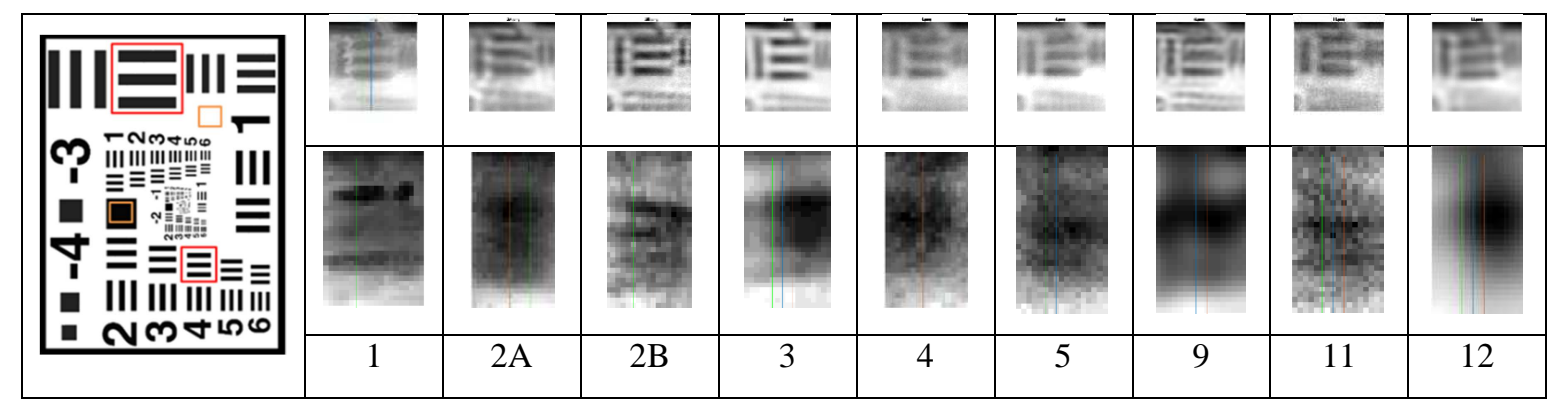

Figure 14: Left: Image region used for evaluation; Right: Evaluated bar patterns for the different images. Top row, demagnified large bar patterns; bottom row, magnified small bar patterns. 
CNR is not exactly the same measure as the algorithm performance evaluation done by the group looking for the smallest resolvable bar pattern on the USAF 1951 test target in each restored submission including smaller bars. However, if we compare the results of CNR on the small bar pattern with the group evaluation, we see that the best image is also image $2 \mathrm{~B}$, followed by image 1 and image 3 .
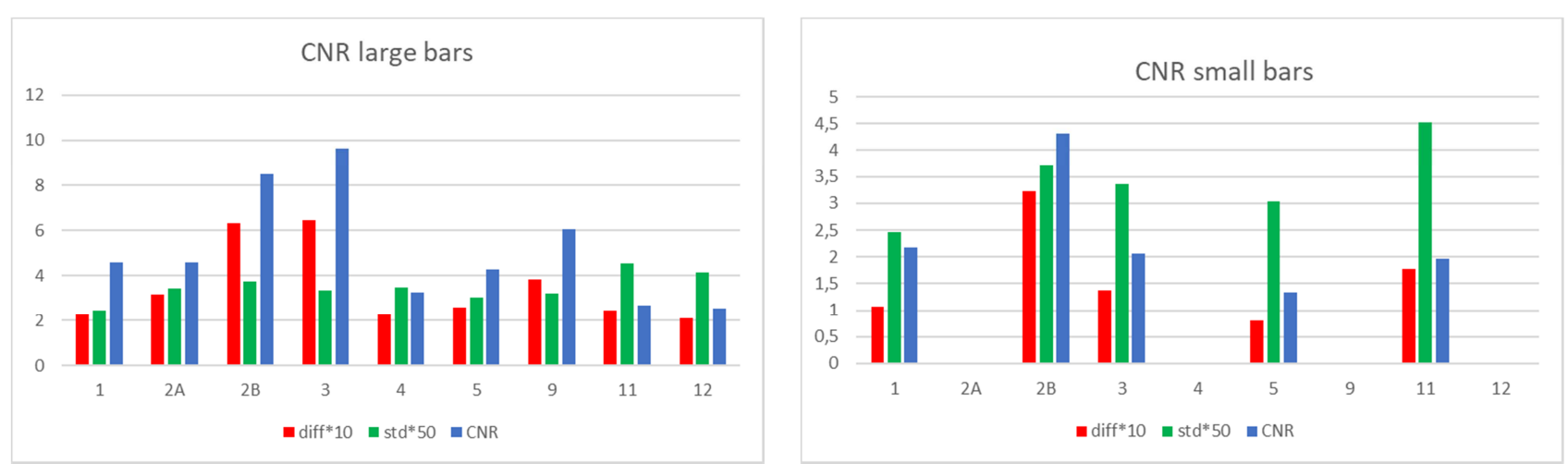

Figure 15: CNR evaluation for the large (left) and small (right) bar values. Note that the difference is multiplied by 10 and the std by 50 to obtain values in the same range as the CNR.

An improvement of this method would be the automatic selection of all bar patterns in the images, and then evaluate either the improvement for all different frequencies, or select the smallest bar pattern for which still three bars can be discerned. For this, the method should also be changed so that the difference value will depend on three bars instead of the maximum and minimum in the area.

\section{CONCLUSION}

During the trial, a large amount of data was recorded under a variety of turbulent conditions in both controllable indoor and natural outdoor environments. A data-base was developed and completed with relevant information (ground truth data, optical sensors characteristics...) to validate theoretical and numerical models.

The images collected were used for a Restoration Challenge that brought together experts in the field and allowed them to process identical input data using state-of-the-art algorithms.

In parallel, new turbulence characterization instruments and methods have been developed and tested in particular to achieve the turbulence profile along the line of sight and have thus extended the path averaged measurement given by current instruments such as the scintillometer or local measurement given by eddy covariance or anemometers sensors.

Emerging capabilities have been tested and evaluated like the plenoptic camera which simultaneously records multiple images of the same object from slightly different angles of view through differing paths in the turbulent atmosphere. Unfortunately, the recorded images were not sufficiently resolved to conclude but this can be further investigated in a follow-on group.

We plan to further develop turbulence characterization methods to provide a simple and effective way to evaluate the actual performance of optical systems during operational use. We also highlighted a lack of relevant metrics to assess the effects of turbulence on system performance and the effectiveness of correction techniques. This point will be addressed in the next group. We will also extend the comparison of image processing algorithms to other turbulence conditions and kind of images in a "Signal Processing Cup Challenge" using the metrics to score the results.

\section{ACKNOWLEDGEMENTS}

The trial was partially supported by NATO RTO SET Panel. The NATO SET 226 field trial was expertly organized and hosted by Jean-Francois Daigle and his colleagues at DRDC Valcatier. Special thanks to Pascal Duchesne and Gaston Nadeau for their support and patience during the trial. Thanks to Lt Matt Trippel (USAF) for enthusiastically serving as camera operator and technician during the field trial. 


\section{REFERENCES}

[1] Smith, Frederick G. "Atmospheric propagation of radiation." The infrared and electro-optical systems handbook, Bellingham: SPIE-The International Society for Optical Engineering and Ann Arbor, Michigan: ERIM-Infrared Information Analysis Center, edited by Acceta, et al. (1993).

[2] Gladysz, S, "Nearly Complete Characterization of Optical Turbulence with an LED Array," in Imaging and Applied Optics 2017 (3D, AIO, COSI, IS, MATH, pcAOP), Optical Society of America, 2017

[3] Chassat, F. «Propagation optique à travers la turbulence atmosphérique. Etude modale de l'anisoplanétisme et application à l'optique adaptative », thèse de doctorat, Université de Paris Sud, Juin 1992

[4] Lumsdaine, A. and T. Georgiev. The focused plenoptic camera. in 2009 IEEE International Conference on Computational Photography (ICCP). 2009.

[5] Wilson, Richard C., and Edwin R. Hancock. "Plenoptic Imaging for Seeing Through Turbulence." Joint IAPR International Workshops on Statistical Techniques in Pattern Recognition (SPR) and Structural and Syntactic Pattern Recognition (SSPR). Springer, Cham, 2018.

[6] $\mathrm{Wu}$, Chensheng, Jonathan Ko, and Christopher C. Davis. "Determining the phase and amplitude distortion of a wavefront using a plenoptic sensor." JOSA A 32.5 (2015): 964-978.

[7] Wu, Chensheng, Jonathan Ko, and Christopher C. Davis, "Imaging through strong turbulence with a light field approach", Optics Express Vol. 24, No. 11, (2016).

[8] Kanaev, Andrey V., et al. "Restoration of images degraded by underwater turbulence using structure tensor oriented image quality (STOIQ) metric." Optics express 23.13 (2015): 17077-17090

[9] Carmen J. Carrano, "Speckle imaging over horizontal paths", Proc. SPIE 4825, High-Resolution Wavefront Control: Methods, Devices, and Applications IV, (6 November 2002)

[10] Eric J. Kelmelis, Stephen T. Kozacik, Aaron L. Paolini, "Practical considerations for real-time turbulence mitigation in long-range imagery," Optical Engineering 56(7)

[11] Hunt, B.R., "Minimizing the Computation Time for Using the Techniques of Sectioning for Digital Filtering of Pictures," IEEE Transactions on Computers, Vol. C-21, pp. 1219-1222, 1972.

[12] Trussell, H.J. and B.R. Hunt, "Image Restoration of Space-Variant Blurs by Sectioned Methods," IEEE Trans., Acoustics, Speech, Signal Processing, Vol. ASSP-26, 1978.

[13] Matson, C. L., Borelli, K., Jefferies, S. M., Hege, E. K., Beckner, C. C., and Lloyd-Hart, M., "A fast and optimal multi-frame blind deconvolution algorithm for high-resolution, ground-based imaging of space objects", Applied Optics, 48, A75-A92 (2009).

[14] Russell C. Hardie, Michael A. Rucci, Alexander J. Dapore, Barry K. Karch, "Block matching and Wiener filtering approach to optical turbulence mitigation and its application to simulated and real imagery with quantitative error analysis," Optical Engineering 56(7)

[15] N. Anantrasirichai, A. Achim, N. G. Kingsbury and D. R. Bull, "Atmospheric Turbulence Mitigation Using Complex Wavelet-Based Fusion," IEEE Transactions on Image Processing, vol. 22, no. 6, pp. 2398-2408, June 2013.

[16] Droege, D. R., Hardie, R. C., Allen, B. S., Dapore, A. J., \& Blevins, J. C. (2012). A real-time atmospheric turbulence mitigation and super-resolution solution for infrared imaging systems. Proceedings of SPIE Defence Security and Sensing. Baltimore, MD.

[17] Chan, Stanley H., Xiran Wang, and Omar A. Elgendy. "Plug-and-Play ADMM for image restoration: Fixed-point convergence and applications." IEEE Transactions on Computational Imaging 3.1 (2017): 84-98.

[18] Zhu, Xiang, and Peyman Milanfar. "Removing atmospheric turbulence via space-invariant deconvolution." IEEE transactions on pattern analysis and machine intelligence 35.1 (2013): 157-170.

[19] Lou, Yifei, et al. "Video Stabilization of Atmospheric Turbulence." Inverse Problems \& Imaging 7.3 (2013) 